\title{
DESCRIPCIÓN DE FACIES DE LA FORMACIÓN SURETKA EN LOS ALREDEDORES DE SIQUIRRES Y SU RELACIÓN CON AMBIENTES DE DEPOSITACIÓN
}

\author{
DESCRIPTION OF FACIES OF THE SURETKA FORMATION NEAR \\ SIQUIRRES AND HIS RELATION WITH DEPOSITIONAL ENVIRONMENTS
}

\author{
Asdrúbal G. Vargas*, Jorge Salazar y Manuel Barrantes \\ Instituto Costarricense de Electricidad (I.C.E.), Costa Rica \\ *Autor para contacto: avargass@ice.go.cr
}

(Recibido: 04/04/2017; aceptado: 06/06/2017)

\begin{abstract}
In this work a description of sedimentary facies identified for the Suretka Formation near the town of Siquirres, Limón, is presented. The description was carried out with the inspections of underground excavations, outcrops in creeks, shafts, and boreholes during the construction stage of the Reventazón Hydroelectric power plant. Three sedimentary facies were identified: conglomerate facies, sandstone lenses with fine to very fine conglomerate and tuff lenses facies. The conglomerate facies is characterized by igneous blocks sumerged in a loam sandy matrix. These facies have metric to decametric bedding with bad gradation. On the other side facies of sandstone lenses and fine conglomerates are described as strata of the centimeter order with presence of both parallel and cross laminations in sandstone lenses and reverse and normal gradations in fine conglomerate lenses. The facies analysis allows the identification of an environment of subareal deposition associated with alluvial fans. For the facies of conglomerates, a proximal alluvial fan environment is established and for the facies of lenses of sandstones and intercalations of fine to very fine conglomerates a environment of medium alluvial fan with meandering sedimentary processes like longitudinal or point bars. Keywords: facies, conglomerate, Suretka Formation, Limón Basin, sedimentation environment.
\end{abstract}

RESUMEN: Se presenta la descripción de facies de la Formación Suretka en los alrededores de la localidad de Siquirres, Limón. Dicha descripción se realizó por medio de la inspección de quebradas, taludes, obras subterráneas y perforaciones realizadas durante la construcción de la planta hidroeléctrica Reventazón. Se identificaron tres facies sedimentarias: facies de conglomerados, facies de lentes de areniscas e intercalaciones de conglomerados finos-muy finos y facies de lentes de toba. Las facies de conglomerado se caracteriza por la presencia de bloques de origen ígneo inmersos en una matriz limo arenosa. Por lo general se presentan estratos métricos hasta decamétricos mal gradados. Por el contrario las facies de lentes de areniscas y conglomerados finos se describen como estratos del orden centimétrico con presencia de laminaciones tanto paralela como cruzada en los lentes de arenisca y gradaciones inversas y normales en los lentes de conglomerado fino. El análisis de las facies permite identificar un ambiente de depositación

Vargas, A.G., Salazar, J. y Barrantes, M. (2017). Descripción de facies de la Formación Suretka en los alrededores de Siquirres y su relación con ambientes de depositación. Revista Geológica de América Central, 57, 85-95. doi: 10.15517/rgac.v0i57.30149 
subáereo asociado a abanicos aluviales.Para las facies de conglomerados se establece un ambiente de abanico aluvial proximal y para las facies de lentes de areniscas e intercalaciones de conglomerados finos-muy finos un ambiente de abanico aluvial medio con procesos sedimentarios meándricos tipo barras longitudinales o de punto.

Palabras clave: facies, conglomerado, Formación Suretka, cuenca Limón, ambiente de sedimentación.

\section{INTRODUCCIÓN}

Sapper (1905) señala la presencia de conglomerados en las cercanías de la localidad llamda Suretka, ) señala la presencia de conglomerados en las cercanías de la Milla 44, mencionando el buzamiento de los conglomerados. Por su parte Berry (1921) lo refiere como conglomerado Suretka. Paris (1953) refería esta formación como predominantemente conglomerática y piroclástica, asociada en su parte basal con coladas y brechas basálticas en el área Reventazón - Chirripó. Dengo (1962) describe una sección vulcanogénica en el Río Pey y en el área de su desembocadura en el Río Telire, consistente en coladas de basaltos afaníticos intercalados con brechas, coladas brechosas y tobas en menor proporción. Por su parte Campos (1987) indica que gradan lateralmente a las limolitas calcáreas, lutitas y calizas arrecifales de la formación Limón de edad Pleistoceno-Holoceno. Rojas (1996) menciona que aflora en los ríos Siquirres, Pacuare, Pacuarito y Cimarrones y en las quebradas La Planta, Cabros y Seca con un espesor medido de $500 \mathrm{~m}$. Otros autores que han realizado investigaciones sobre esta formación son Cervantes y Soto (1988). Por su parte Bottazzi (2016, pág. 32) describe a la formación Suretka como conglomerados y brechas polimícticas (de diferente composición) y areniscas medias con cemento silicio dispuestos en bancos de decenas de metros de espesor asociados a un ambiente aluvial. Su contacto inferior es transicional con la formación Río Banano y discordante con las formaciones Senosri y Uscari. Se interdigita con las formaciones volcanogénicas del Cuaternario (Bottazzi et al, 1994).

El objetivo del presente trabajo es la identificación de facies sedimentarias de la formación Suretka incluyendo: descripción litológica, estructuras sedimentarias, caracterización petrográfica y correlaciones estratigráficas para su respectivo análisis y discusión con respecto a su génesis y contexto tectónico-sedimentario de depositación en los alrededores de Siquirres cuenca de Limón Sur. El área de estudio abarcó los alrededores del Río Reventazón desde el sitio de presa de la Planta Hidroeléctrica Reventazón hasta la intersección del río con la ruta 32, así como diferentes quebradas afluentes al río como lo son la quebrada Rubio, Borrachos, Tres Amigos, Casa de Máquinas y Guayacán. (Fig. 1). Esta investigación se restringe a la descripción de las facies de la formación Suretka de forma local para el área de estudio. La metodología de trabajo consistió en la revisión de antecedentes en la literatura incluyendo la Revista Geológica de América Central (por ejemplo Denyer et al.,1987) e informes internos del Instituto Costarricense de Electricidad (Cerdas, 2000; Arias, 2007), el estudio de afloramientos de roca en galerías, excavaciones y quebradas, así como el estudio de los núcleos de roca en perforaciones de tipo sacanúcleos realizadas como parte de la construcción de la Planta Hidroeléctrica Reventazón.

\section{FACIES}

De forma general se identificaron un total de 3 facies en los diferentes sectores estudiados.

\section{Facies de Conglomerado}

Corresponden con la presencia de bloques redondeados a subredondeados inmersos en una matriz limo arenosa de color café. Se incluyen 


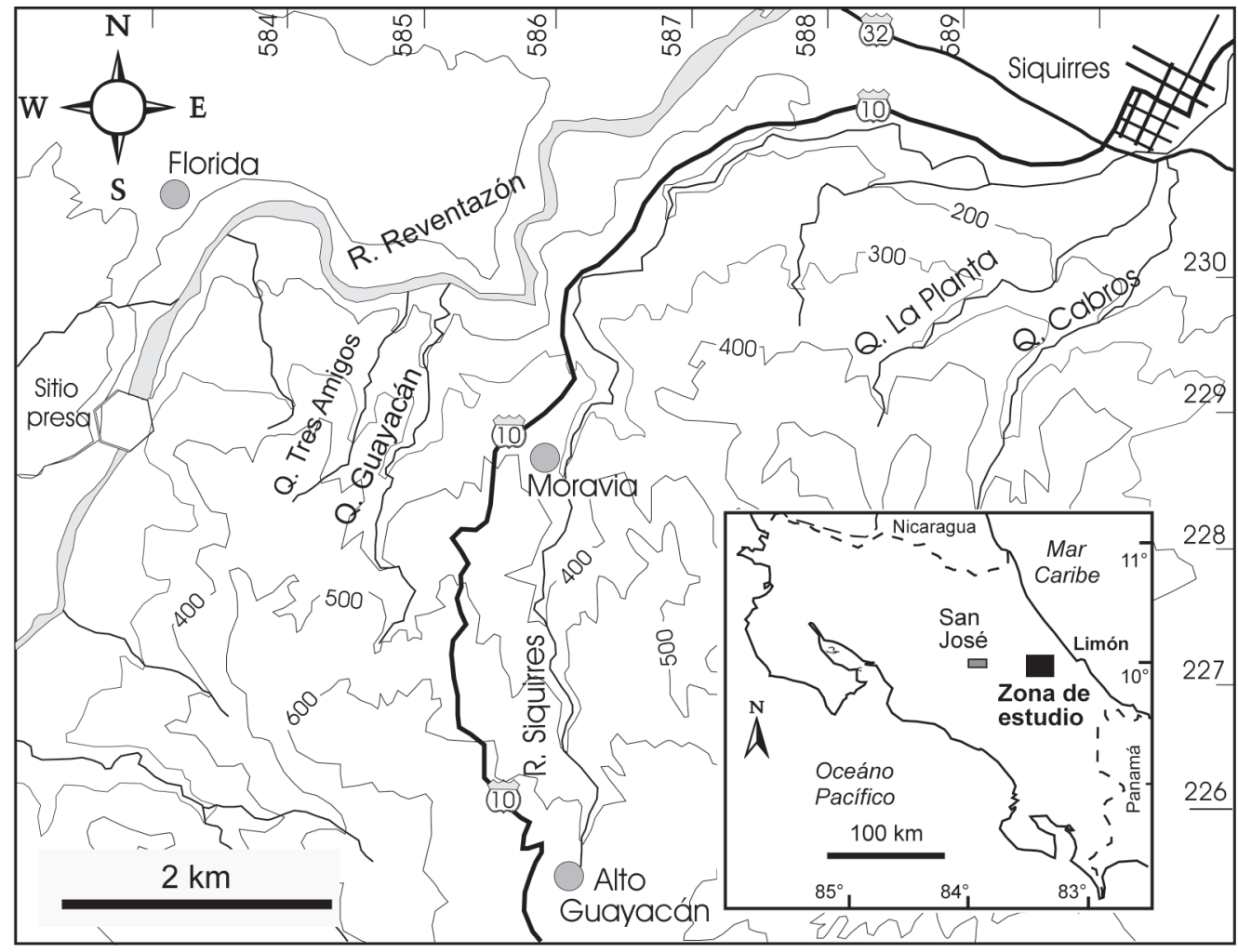

Fig. 1: Ubicación del área de estudio de la formación Suretka.

conglomerados gruesos compuesto por bloques con tamaño variable entre 1 y $4 \mathrm{~m}$ y conglomerados medios compuesto por bloques con tamaño variable entre $15 \mathrm{~cm}$ y $1 \mathrm{~m}$ (Fig. $2 \mathrm{~A}$ y $2 \mathrm{~B}$ ).

La matriz en la mayor parte de los casos representa entre un 20 y un $40 \%$ del volumen y se compone principalmente de arena gruesa a limos y arcillas. Microscópicamente se destaca la presencia de plagioclasas, piroxenos, hornblenda verde, olivinos, los cuales corresponden con la misma génesis de los clastos volcánicos del conglomerado. Adicionalmente en la matriz se nota la presencia de arcilla (15-20\%) de color café, la cual contribuye a la adherencia entre los granos de la matriz, no se identificó presencia de cemento silicio, el cual es mencionado por otros autores. Está ausencia de cemento estaría relacionada con el ambiente de depositación de la facie que se analiza en detalle en el apartado de discusión. Los líticos existentes son de composición variada, sobresalen líticos volcánicos (LV en Fig. 3A) y con formas redondeadas, flotando en una matriz fina parda con presencia de cristales. (Fig. 3B). También es común la presencia de abundantes fragmentos líticos volcánicos típicamente andesíticos en algunas ocasiones con cierto grado de alteración. (Fig. 3 C), de textura porfirítica con fenocristales idiomórficos de plagioclasa, augitas y opacos en una mesostaza hipocristalina rica en opacos finos y microlitos de plagioclasa. También se logran identificar fragmentos líticos de basaltos olivínicos alcalinos, típicos de la Formación Guayacán (Fig. 3D). Otro tipo de fragmento lítico presente parece corresponder a intrusivos de carácter más bien básico dada su composición mineralógica y sus rasgos texturales.

El contacto entre bloques varía entre contactos planares, puntuales y bloques flotantes, lo que se evidencia a lo largo de la formación como estratos de conglomerados soportados por matriz así como soportados por clastos entre ellos. Con base en Boggs (2009) petrológicamente se 
caracterizan como conglomerados epiclásticos extraformacionales y dado que la mayor parte de bloques corresponden a una misma litología pueden ser clasificados como conglomerados monomícticos, más detalladamente a partir de la composición de sus clastos clasifican como conglomerados petromícticos andesíticos-basálticos.

La principal estructura sedimentaria dentro de las facies de conglomerado corresponde a la estratificación (Fig. 4A). Esta varía significativamente a lo largo de la formación, registrándose intercalaciones centimétricas $(10$ a $30 \mathrm{~cm})$ de estratos de conglomerados con contactos bien definidos hasta estratos métricos y decamétricos de conglomerado de apariencia masiva y mal graduados. A lo largo de la formación es común la presencia de estructuras registradas como lentes de guijarros (sin matriz) asociados a algún proceso de depositación particular. De igual manera es posible observar gradaciones normales e inversas en los depósitos de conglomerados. Otra estructura identificada en varios afloramientos expuestos en taludes labrados en forma natural por la acción erosiva del río Reventazón es la orientación de clastos elongados (Fig. 4B). De forma general la dirección de buzamiento de estas facies en la zona de estudio es hacia el NW con ángulos de buzamiento variable entre $15^{\circ}$ y $20^{\circ}$.

\section{Facies de lentes de arenas e intercalaciones de conglomerados finos-muy finos.}

Se incluyen:

- Conglomerado finos compuestos por guijarros de tamaño variable entre $5 \mathrm{~cm}$ y $15 \mathrm{~cm}$. (Fig. 5A).

- Conglomerado muy fino compuesto por guijarros de tamaño variable menores a $5 \mathrm{~cm}$ (Fig. 5B) y lentes de arenas que varían en su grado de litificación, así como en su espesor principalmente entre 0,15 y $3,6 \mathrm{~m}$ y su extensión lateral entre 1 y $30 \mathrm{~m}$. Se identificaron tanto lentes muy constantes dentro la secuencia estratigráfica hasta estructuras de pocos metros (Fig. 5C). Los lentes con menor grado de litificación suelen tener extensiones y espesores menores correspondiendo a depositaciones muy localizadas (Fig. 5D).

Se identifican tanto laminaciones paralelas (Fig. 6A) como cruzadas (Fig. 6B) en los diferentes lentes de arena. Los conglomerados finos $\mathrm{y}$ muy finos suelen presentar gradaciones inversas y normales.

\section{Facies de lentes de toba}

Los lentes de toba se presentan con una menor frecuencia que los lentes de areniscas, con espesores de entre $0,5 \mathrm{~cm}$ y $1 \mathrm{~m}$ y extensiones decimétricas.

\section{RELACIONES ESTRATIGRÁFICAS}

En los alrededores de Siquirres la formación Suretka sobreyace a los flujos lávicos de la formación Guayacán, el contacto es relativamente concordante con cierto grado de irregularidad y erosión (Fig. 8A). Además se identificó el contacto entre una lava alterada y el conglomerado marcando la etapa final del vulcanismo de la formación Guayacán (Fig. 8B) y el inicio de la depositación de la formación Suretka. Varios afloramientos de este contacto se identificaron en galerías de investigación del sitio de presa Reventazón. Por su parte el contacto superior de la unidad se presenta con los debris avalanche asociados a la formación Doán (Fig. 8C). Por la génesis de formación del debris avalanche, al registrarse evidencia de rampas de avance del movimiento de estos flujos se observa como el mismo erosionó e inclusive incorporó fragmentos métricos de conglomerado al debris avalanche (Fig. 8D). Particularmente en el área de estudio no se observó contacto de la formación Suretka con rocas sedimentarias más antiguas de la cuenca de Limón Sur como es el caso de la formación Río Banano y Uscari. El espesor total medido de la formación en el área de estudio varía entre $90 \mathrm{~m}$ y $120 \mathrm{~m}$. 

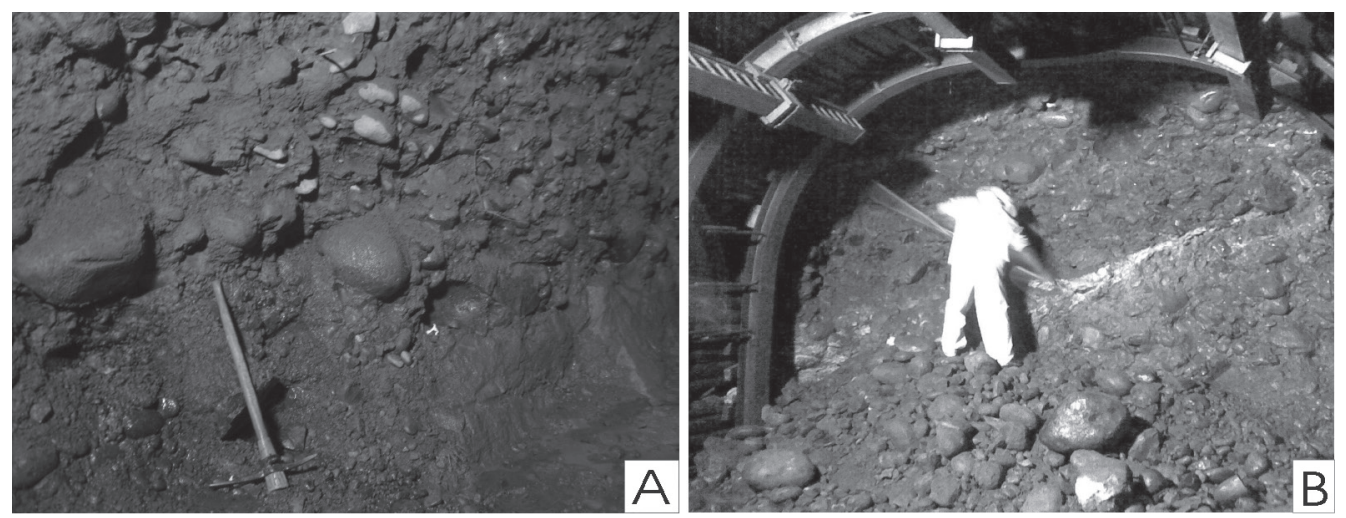

Fig. 2A: Conglomerado grueso. 2B Conglomerado medio.

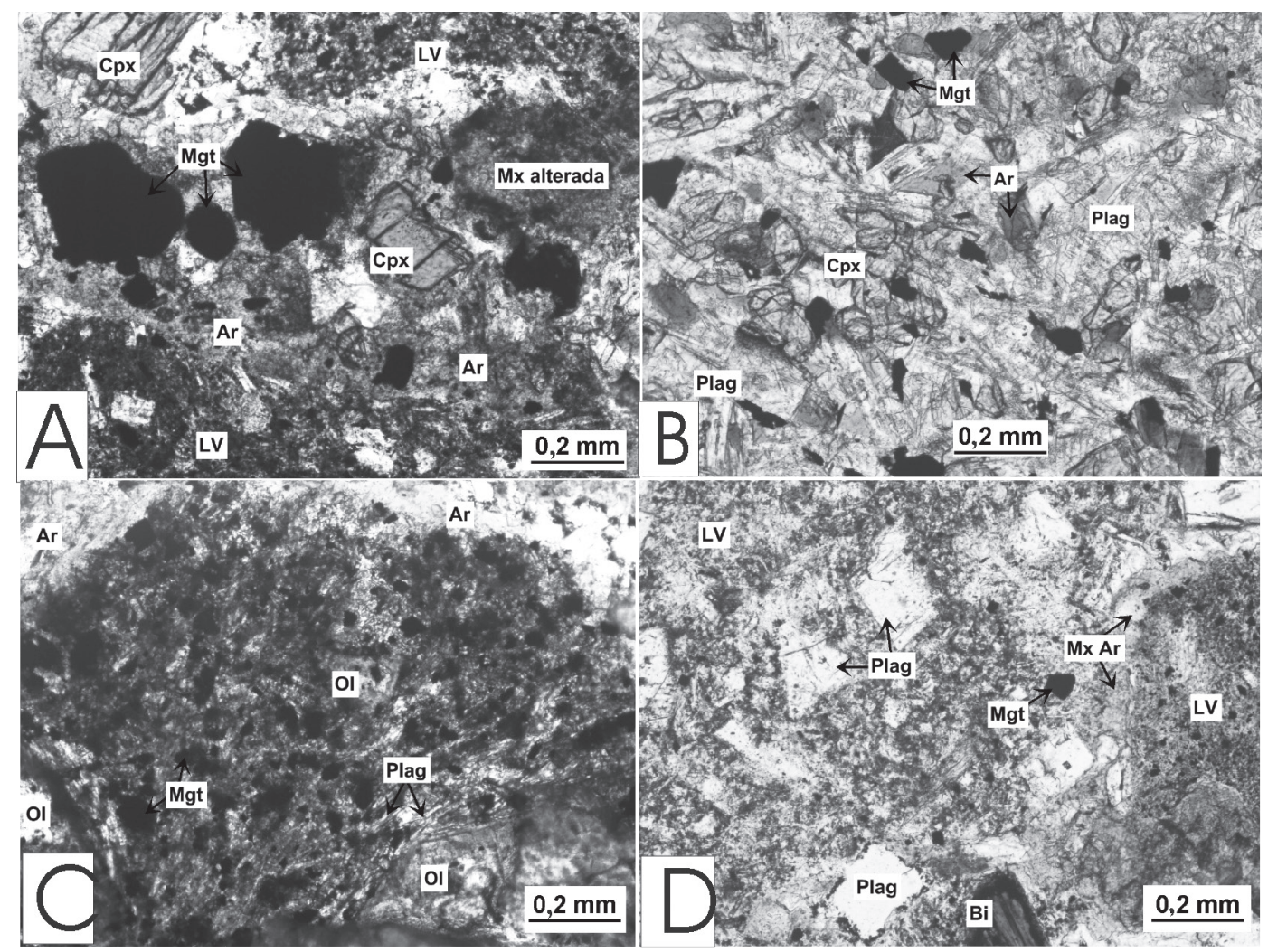

Fig 3A: A) Fragmentos líticos volcánicos (LV) de color pardo oscuro con presencia de cristales de clinopiroxenos (Cpx) y magnetita (Mgt) con matriz alterada (Mx alterada), dentro de una matriz fina y pequeñas oquedades rellenas parcialmente de arcillas (Ar) B). B) Fragmento de textura holocristalina-intergranular hasta subofítica y con cristales de plagioclasa (Plag), magnetita (Mgt), clinopiroxeno (Cpx). Algunos minerales están transformados a arcillas (Ar). C). Fragmento lítico de un basalto alcalino (Fm. Guayacán) con cristales de olivino iddingsitizado (Ol), plagioclasas (Plg) y magnetita (Mgt). Alrededor hay presencia de arcillas (Ar). D). Fragmento lítico andesítico (LV) con textura porfirítica, abundantes fenocristales de plagioclasa (Plag), magnetita (Mgt), biotita (Bi) y matriz arcillosa (MxAr) 

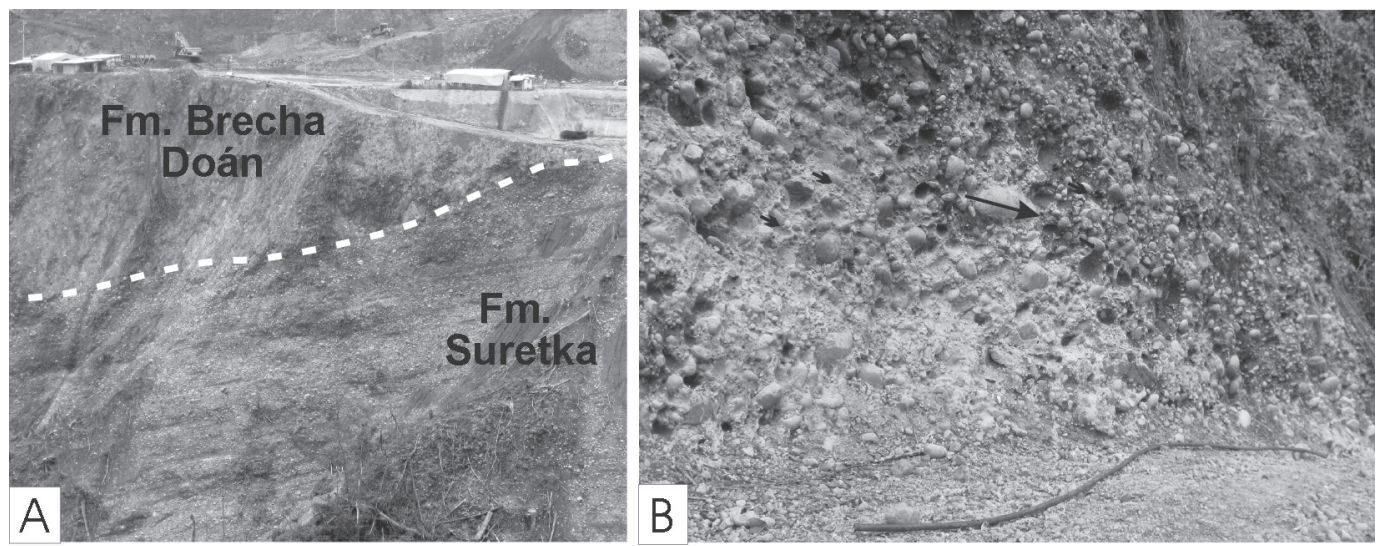

Fig. 4: A) Estratificación en el conglomerado en el talud de margen derecha del sitio de presa Reventazón. B) Orientación de clastos en el talud de margen izquierda del sitio de presa.
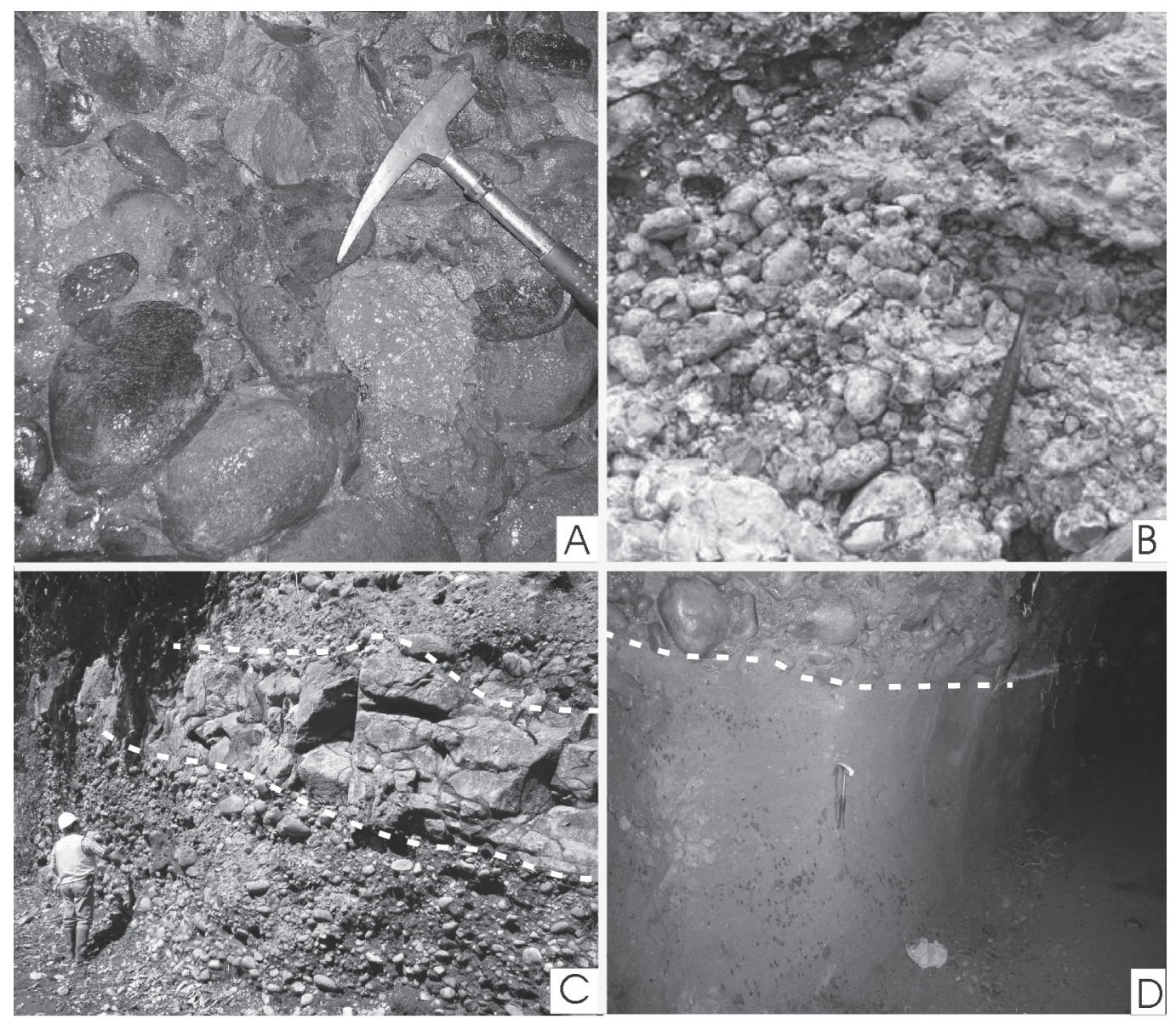

Fig. 5A: Conglomerado fino con escaza cantidad de matriz. B) Conglomerado muy fino con gradación inversa hacia un conglomerado medio. C) Lente de arenisca en talud MI del sitio de presa del PHR de 1,5 m de espesor. D) Lente de arenisca menos litificado. 

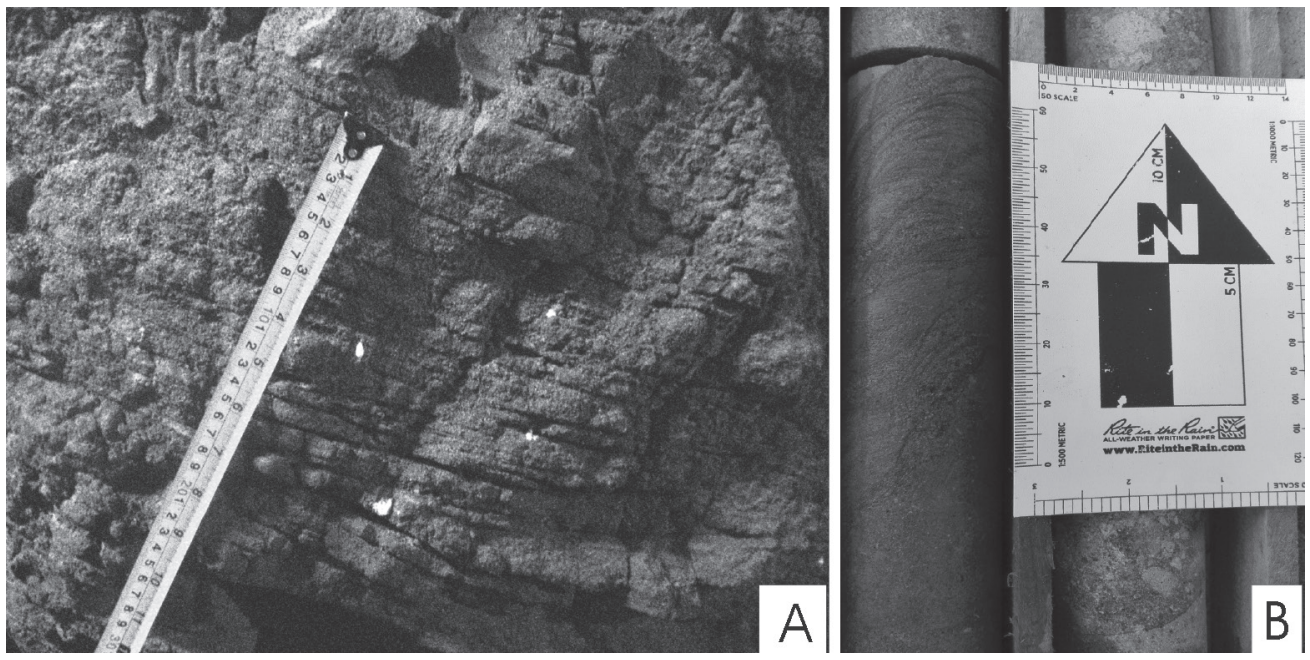

Fig. 6A: Laminación paralela en lente de arenisca en la Galería 140 MI de la planta hidroeléctrica Reventazón. 6B) Laminación cruzada en núcleo de perforación de lente de arenisca.

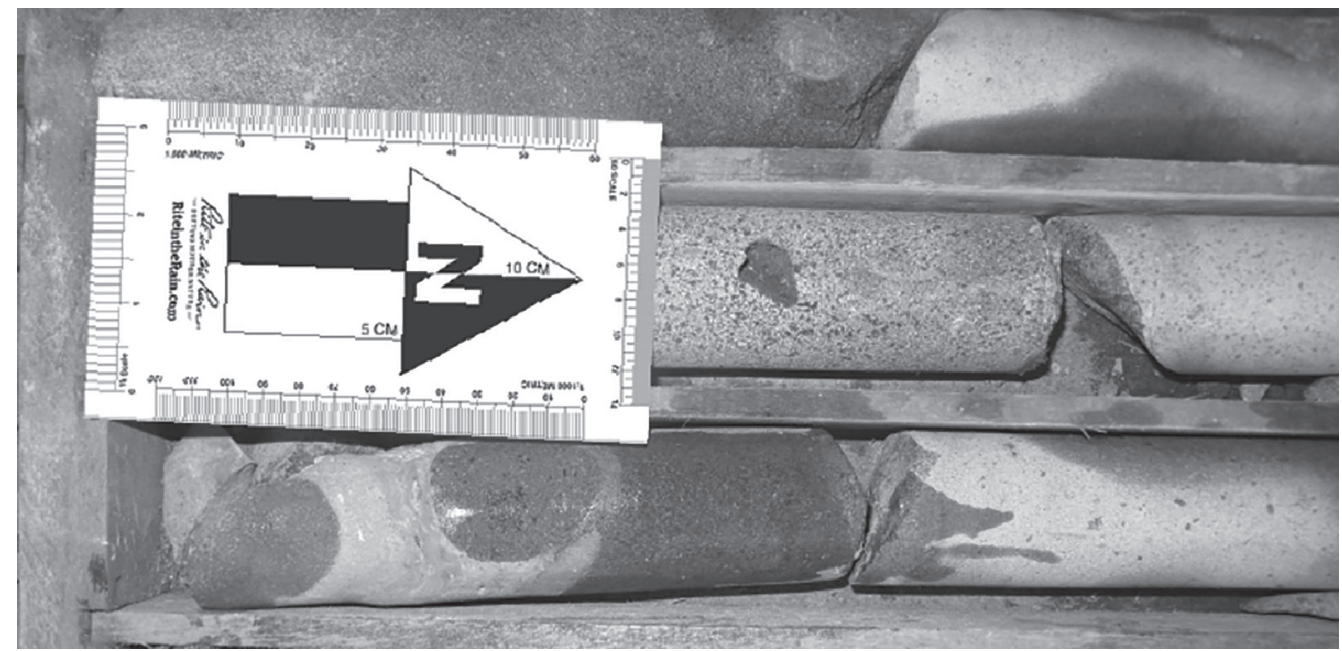

Fig. 7: Lente de toba en núcleo de perforación.

\section{DISCUSIÓN}

Campos (1987), ha interpretado estos depósitos como depósitos de fan delta. Botazzi et al (1994) identificaron facies de tipo estuario y fluvial. Taylor (1975), Campos (1987) y Botazzi et al., (1994) le atribuyen una edad Plioceno Tardío a Reciente. Según Linkimer y Aguilar en Denyer y Kussmaul; 2000, estas rocas indican el inicio de una sedimentación en un ambiente continental y su depositación está relacionada a la erosión intensa de materiales volcánicos y transporte de rocas de la Cordillera de Talamanca debido al levantamiento de la misma partir del MiocenoSuperior, acumulándose espesores de hasta 2000 $\mathrm{m}$ de sedimentos de tipo aluvial y coluvial al pie de la cordillera (Denyer, Alvarado y Aguilar 2000 en Denyer y Kussmaul, 2000). 

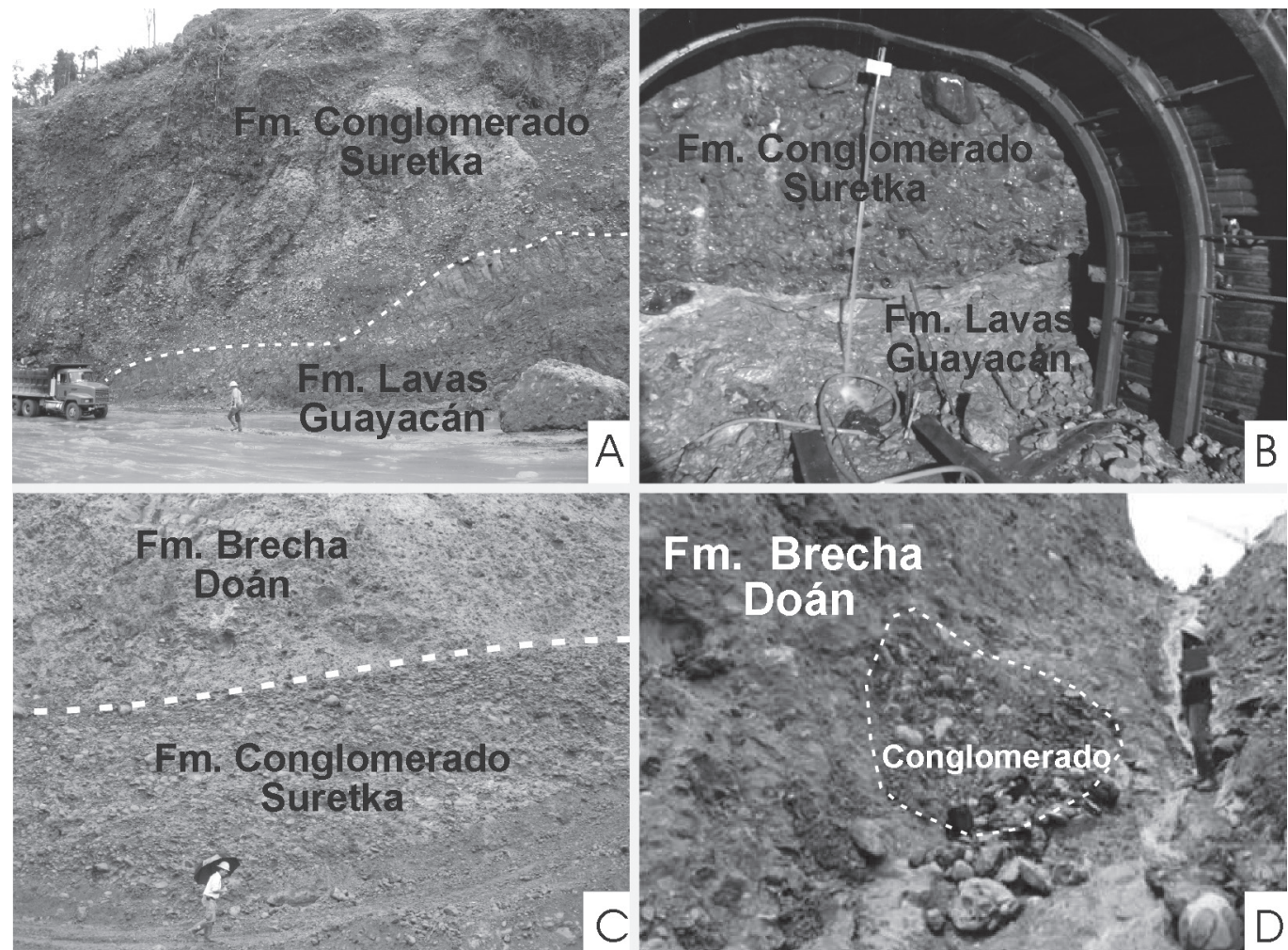

Fig. 7: A) y B) Contacto inferior de Fm Suretka con Fm Guayacán en excavaciones subterráneas y taludes del sitio de presa de la planta hidroeléctrica Reventazón. C) Contacto superior con Fm Doán en talud de vertedero de excedencias de planta hidroeléctrica Reventazón. D) Bloque de conglomerado inmerso en la formación Doán.

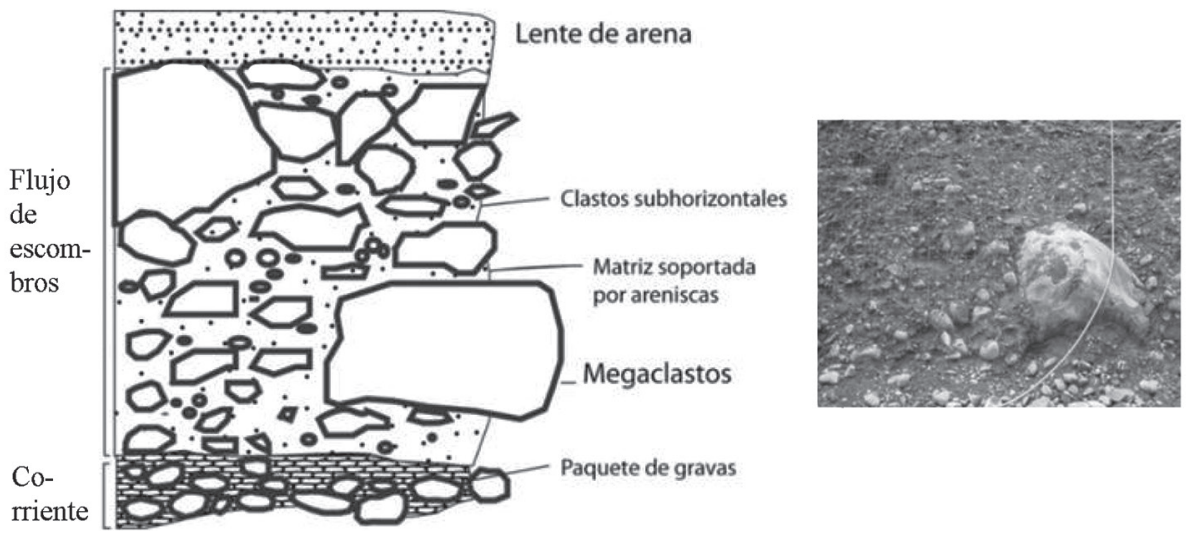

Fig. 8: A) Parte de columna estratigráfica típica de un ambiente de depositación subáereo donde destacan grande bloques soportados por matriz y mal graduados. (Modificado de Boggs, 2009). B) Fotografía de facie de conglomerado donde se aprecia un depósito mal graduado con megabloques correlacionable a un ambiente de depositación subáereo. 

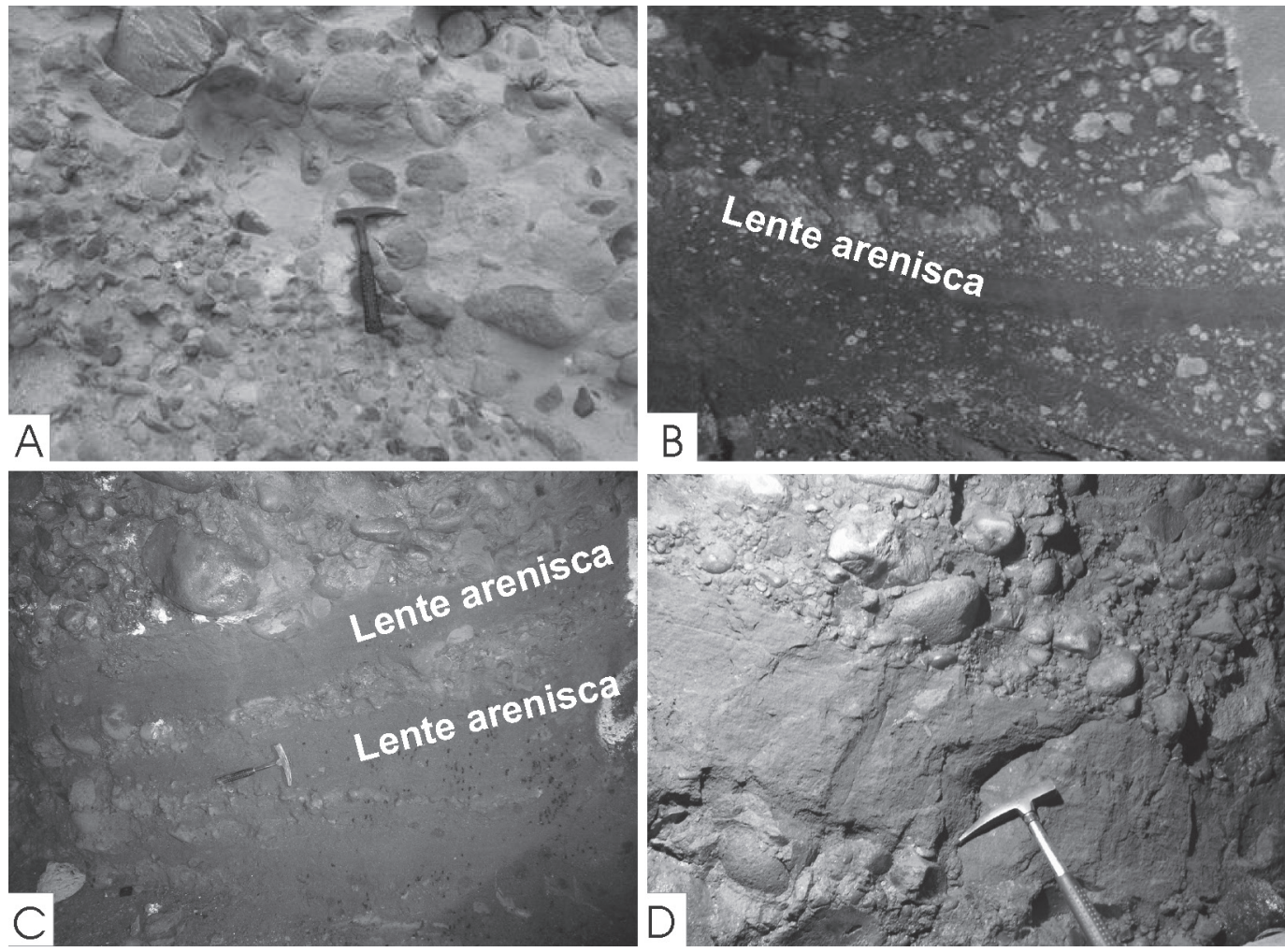

Fig. 9: A) Gradación inversa facies de conglomerados finos a medios. B) C) y D) Facies de areniscas intercalados con depósitos de guijarros correspondiente a un ambiente de abanico aluvial en su parte media con presencia de barras longitudinales o de punto en un sistema meándrico.

A partir del análisis de las facies principalmente de conglomerados es posible determinar un ambiente de depositación subaéreo para la formación Suretka asociado a flujos de detritos y abanicos aluviales. En este ambiente es común la presencia de bloques de gran escala, soportados por matriz y mal graduados (Figs.9 A y B).

De igual manera es común la presencia de gradaciones inversas y estratificación poco desarrollada con delgadas capas de arenas intercaladas (Fig. 10A). Bottazzi (2016) describe para la cuenca de Limón Sur específicamente para las sucesiones sedimentarias continentales facies de conglomerados como parte de ambientes de abanicos aluviales proximales con características similares a las facies de conglomerados descritas en esta investigación.
Las facies asociadas a zonas de lentes de areniscas con presencia de estratificaciones más finas (conglomerados finos) corresponden con facies asociados probablemente a zonas de canales (ambiente fluvial) por los cuales fluyeron ríos durante la depositación regional de estos abanicos aluviales. Esto coincide con diferentes autores tales como Fernández et. al. (1994) el cual menciona que la formación Suretka se encuentra en la megasecuencia $\mathrm{D}$, que representa el último relleno de la cuenca en donde los sedimentos más jóvenes de esta secuencia son depósitos progradantes de abanicos aluviales de edad Plio-Pleistoceno. De igual manera Bottazzi (2016) describe zonas de abanico aluviales medios con características de procesos 
sedimentarios meándricos con formación de barras longitudinales o de punto que coinciden de buena manera con lo descrito para las facies de lentes de arena e intercalaciones de conglomerados finos de esta investigación.

Adicionalmente se interpreta que en los períodos de depositación de las facies sedimentarias de la formación de Suretka, los canales fluviales distribuían los materiales arrastrados desde las áreas fuente (zonas montañosas de erosión) y cada vez que acontecían eventos de gran energía asociados a períodos de abundantes lluvias, ocurría la depositación enérgica y caótica de flujos de detritos redondeados de tamaño métrico a decimétrico. Por su parte en los períodos intermedios, de mayor tranquilidad climatológica, dominaba la sedimentación caracterizada por granulometrías finas y más retrabajadas, típicas de los depósitos de canal (Fig. 10 B, C y D). Los cuales podían ser totalmente erosionados durante el siguiente episodio de depositación masiva o en algunos casos ocurrió una erosión parcial y sepultamiento de los mismos quedando integrados a la secuencia sedimentaria. De igual manera se interpreta que durante estos periodos de calma coexistía de manera ocasional una actividad volcánica que permitió la sedimentación de las facies relacionadas con lentes de tobas.

Con respecto al ambiente tectónico de sedimentación se concuerda con los expuesto por Brandes et al. (2007a y 2007b) quienes interpretan que la depositación de estos conglomerados se llevó a cabo en cuencas de tipo "piggy back basin" desarrolladas como consecuencia de un esfuerzo compresivo que ha variado muy poco tal como lo indican Denyer et al. (1994) desde el paleógeno hasta el cuaternario.

Lo anterior en desacuerdo con lo mencionado por Cervantes y Soto (1988) los cuales mencionan un régimen distensivo en el sector de Siquirres, contrario a lo analizado en este estudio donde se evidencia notoriamente un patrón compresivo en el área que inclusive afecta con desplazamientos inversos y estructuras de pliegues a la formación Suretka y Dóan. Este patrón tal como lo menciona Campos (1987) es probable que varié a un sistema de fallas transcurrentes hacia la parte media-alta de la cuenca en donde los macizos volcánicos em- piezan a tener una influencia importante en la variación de la orientación de esfuerzos. Finalmente se concluye dentro de un contexto tectónico y geológico regional que los facies analizadas corresponden a la zona de transición entre la sección alta y media de un abanico aluvial.

\section{REFERENCIAS BIBLIOGRÁFICAS}

Arias, O. (2007). Informe de avance a la factibilidad del proyecto Reventazón. San José: ICE. Informe interno.

Berry, E.W., 1921: Tertiary Fossil Plants from Costa Rica. Proc. U.S. National Museum, 59( 2367), 169-185.

Botazzi, G. (2016). Tratado de sedimentología de la cuenca Limón Sur-Costa Rica. Evolución tectono-sedimentaria, secuencias y reconstrucción paleogeográfica (Tesis de licenciatura inédita). Universidad de Costa Rica, San José, Costa Rica.

Botazzi, G., Fernández, J. A. y Barboza, G. (1994). Sedimentología e historia tectono-sedimentaria de la cuenca Limón Sur. Profil, 7: 351-391.

Brandes, C., Astorga, A., Blisniuk, P., Littkes, R. y Winsemann, J. (2007a). Anatomy of anticlines, piggy-back basins and growth strata: a case study from the Limón fold-and-thrust belt, Costa Rica. En G. Nichols, E. Williams y C. Paola (eds), Sedimentary Processes, Environments and Basins: A Tribute to Peter Friend (pp. 91110).Oxford: Blackwell Publishing Ltd.

Brandes, C., Astorga, A., Back, S., Littke, R. y Winsemann, J. (2007b). Deformation style and basin-fill architecture of the offshore Limón back-arc basin (Costa Rica). Marine and Petroleum Geology, 24, 277-287.

Boggs, S. (2009). Petrology of sedimentary rocks. New York: Cambridge Univiversity Press. 
Campos, L. (1987). Geología de la Fila Asunción y zonas aledañas, Atlántico Central de Costa Rica (Tesis de licenciatura inédita). Universidad de Costa Rica, San José, Costa Rica.

Cerdas, A. (2000). Informe de reconocimiento geológico del P. H. Reventazón. San José: ICE. Informe interno.

Cervantez, J. F. y Soto, G. J. (1988). Sedimentación y volcanismo tras-arco Plio Cuaternarios en el área de Siquirres, Costa Rica. Ciencia y Tecnología, 12(1-2), 19-26.

Dengo, G. (1962). Tectonic-igneous sequence in Costa Rica. En A. E. J. Engel, H. J. James y B. F. Leonard (eds), A volume to honor A.F. Buddington (pp. 133-161). Geological Society of Amererica.

Denyer, P., Alvarado, G. y Aguilar, T. (2000). Historia geológica. En P. Denyer y S. Kussmaul (eds), Geología de Costa Rica (pp. 155-167). Cartago: Editorial Tecnológica de Costa Rica.

Denyer, P., Arias, O. y Arias, M. (1994). Esfuerzos y Paleo-esfuerzos de la cuenca de Limón. Revista Geológica de América Central, Volumen Especial Terremoto de Limón, 53-60.

Denyer, P., Feoli, S., Murillo, G. y Rodríguez, C. (1987). Cartografía geológica de un sector de los alrededores de la cuenca alta del río Niñey. Limón, Costa Rica. Revista Geológica de América Central, 7, 113-141.

Fernández, J. A., Bottazzi, G., Barboza, G. y Astorga, A. (1994). Tectónica y estratigrafía de la cuenca Limón Sur. Revista Geológica de América Central, Volumen Especial Terremoto de Limón, 15-28.

Linkimer, L. y Aguilar T. (2000). Estratigrafía sedimentaria. En P. Denyer y S. Kussmaul (eds), Geología de Costa Rica (pp. 155167). Cartago: Editorial Tecnológica de Costa Rica.

Paris, P.H., 1953: Geological Report GRCR-23. San José. Compañía Petrolera de Costa Rica. Inf. interno.

Rojas, M. (1996). Estudio geológico-geotécnico del corredor Turrialba-Siquirres. Sección alto Guayacán-Siquirres del Poliducto Recope. San José: Universidad de Costa Rica. Informe de campaña geológica.

Sapper, K. (1905). Gebirgsbau und boden des südlichen Mittelamerika. Justus Perthes, Gotha, 151: 1-82.

Taylor, G. D. (1975). The geology of the Limón area of Costa Rica (Tesis de doctorado inédita). Universidad de Lousiana, Lousiana, Estados Unidos. 
